Nuclear Physics A471 (1987) 501-519

North-Holland, Amsterdam

\title{
GENERALIZED LIQUID DROP MODEL AND DESATURATION EFFECTS IN NUCLEI
}

\author{
W. STOCKER \\ Universität München, Sektion Physik, D8046 Garching, West Germany \\ P. GLEISSL and M. BRACK \\ Universität Regensburg, Institut für Theoretische Physik, D8400 Regensburg, West Germany
}

Received 6 January 1987

\begin{abstract}
We present a careful analysis of desaturation effects in densities and energies of nuclei using realistic Skyrme interactions in the extended Thomas-Fermi approach. Variational calculations confirm the slab model prescription introduced earlier for the extraction of desaturation terms for spherical systems. Desaturation is found to be appreciable in densities for $A \leqslant 40$ and in energies for $A \leqslant 4$.
\end{abstract}

\section{Introduction}

In contrast to its satisfactory description of nuclear masses, the droplet model $(D M)^{1}$ ) fails to reproduce nuclear radii following from Hartree-Fock (HF) or extended Thomas-Fermi (ETF) methods. The DM radius constant $r_{0}$ was found in ref. $^{2}$ ) not to agree with the HF value. Core densities of heavy nuclei following from the HF-Strutinsky prescription could not be described by the DM approach ${ }^{3}$ ). It is argued in ref. $^{4}$ ) that the DM framework with its schematic treatment of the nuclear surface is too restricted for a satisfactory description of nuclear densities.

The structure of light nuclei should exhibit effects due to the deviation from saturation. The macroscopic DM approach which assumes a saturated structure with a core part separated from a thin (leptodermous) surface is not able to incorporate desaturation. Thus, $\mathrm{HF}^{5}$ ) and $\mathrm{ETF}^{6,7}$ ) nuclear core densities evidently deviate from the DM predictions for light nuclei. In particular, the bending-down of the HF and ETF curves for the relative difference $\delta \rho / \rho_{\infty}$ between central density $\rho_{0}$ and nuclear matter density $\rho_{\infty}$ as a function of $A^{-1 / 3}$ cannot be obtained in the standard DM. Moreover, it could be shown ${ }^{5}$ ) that even all higher order corrections to $\delta \rho / \rho_{\infty}$ obtained in the framework of a generalized liquid drop model (LDM) approach are not able to cause this bending-down of the $\delta \rho / \rho_{\infty}$ curve.

There is a phenomenological folding procedure, the Krappe-Nix method ${ }^{8}$ ) which successfully generalizes the DM for the inclusion of effects following from the finite 
surface thickness and which in principle also is suited to account for desaturation effects.

For a self-consistent treatment of the desaturation effects in densities and energies of small nuclei, one can use the model of a nuclear slab of finite thickness because it allows to study the pure desaturation terms isolated from other terms also occurring in properties of small nuclei, such as e.g. curvature effects. Using this model it was found ${ }^{9,10}$ ) that desaturation sets in below some critical value of the slab thickness which depends on the range of the effective nucleon-nucleon interaction. The desaturation effects decrease exponentially if this critical thickness is increased.

Two attempts can be found in the literature ${ }^{11,12}$ ) which report on mass formula fits supplemented by the inclusion of exponential terms proportional to $\exp \left(-\gamma A^{1 / 3}\right)$ in order to account for desaturation effects. The results, however, do not allow unique conclusions about the importance of such additional desaturation terms in the energy expression.

In ref. ${ }^{13}$ ) a theoretical analysis of desaturation terms in densities and energies of light nuclei was presented starting from slabs as model nuclei where desaturation terms can be studied separately from other effects. A prescription was worked out in this reference which allows to calculate properties of spherical nuclei using the slab results as a basis. Within this framework pure sphericity (or curvature) effects are included, but not effects due to the compression of the nucleus by the surface tension. For various schematic nucleon-nucleon interactions the desaturation effects turned out to depend on the functional form of the interaction, even if the same DM parameters were reproduced. This shows that desaturation represents a new degree of freedom independent of the structure of saturated nuclei. Another result of ref. ${ }^{13}$ ) was that densities are much more affected by desaturation than energies. This was explained as a consequence of the stationarity of the energy with respect to density changes.

In the present paper we report on a variational calculation of spherical $N=Z$ nuclei neglecting the Coulomb interaction. As a realistic effective nucleon-nucleon interaction we favour the $\mathbf{S k M}^{*}$ interaction which reproduces empirical masses, densities and fission barriers excellently ${ }^{6}$ ). The variational ETF approach is practically equivalent to $\mathrm{HF}$ without shell effects ${ }^{6}$ ). The results of the variational calculations for $\delta \rho / \rho_{\infty}$ and the energies are then analyzed with respect to the extraction of desaturation contributions. In order to separate out a desaturation term in the energies we subtract from the calculated energies the generalized liquid drop model (LDM) contribution, which can be independently obtained from a semi-infinite nuclear matter calculation. On the other hand, the results of the variational calculation are used in order to test and confirm the slab model approach of ref. ${ }^{13}$ ). The latter can then be used to study the pure desaturation effects.

We finally conclude that the realistic $\mathrm{SkM}^{*}$ interaction predicts desaturation effects in core densities of nuclei for $A$ less than about 40-50. For the description 
of energies of nuclei, however, desaturation is negligible even for such light systems as $A \cong 6-10$.

\section{Realistic Skyrme interactions and nuclear desaturation}

The expression for nuclear density and energy in terms of the particle number $A$ following from the schematic model and the expansion of the energy outlined in the appendix provide some insight into the structure of LDM and desaturation terms including their coupling in the case of small values of $A$. The possibility to add - in a kind of perturbation approach - desaturation terms independently to the basic LDM contributions, is restricted to the region of $A$ where the desaturation correction is small. For very small nuclear systems a separation of nuclear properties into LDM and desaturation parts is artificial since those nuclei do not exhibit any leptodermous structure.

Thus, basic questions which arise concern (i) the critical mass numbers - different for energy and density - below which desaturation is appreciable and (ii) the mass numbers - even smaller than those - below which the independent addition of LDM and desaturation terms no more reproduces the relevant nuclear quantities.

In this section we shall describe calculations performed using realistic Skyrme interactions in order to obtain answers to these questions. We start from the variational extended Thomas-Fermi method (ETF) ${ }^{6}$ ) as an approximation scheme averaging over shell fluctuations which would disturb the desaturation effects we are interested in.

The use of the Skyrme-ETF procedure for the determination of desaturation needs some justification. Desaturation depends sensitively on the range of the effective two-nucleon interaction. This might be seen from the fact that small systems give up a saturated structure in favour of a more diffuse one where the finite range nucleon-nucleon attraction can work more efficiently. Now, Skyrme forces ${ }^{14}$ ) are often called zero-range forces in contrast to truly finite range forces such as e.g. the Gogny force ${ }^{15}$ ). However, the momentum dependent parts of the Skyrme ansatz lead to an energy density with gradient terms of the density mocking up a finite range of the Skyrme force. Thus, the Skyrme force effectively does have a finite range, and only the question is open whether the Skyrme ansatz reproduces in a reliable way also the special functional form of the two-nucleon interaction.

Normally, the Skyrme-ETF method is presented as a mean-field approach, and one is encountered with the question whether this approach is applicable to small nuclei. We are, however, freed from the discussion of this problem if we follow the spirit of the Hohenberg-Kohn theorem ${ }^{16}$ ). Thereby, we accept the Skyrme-ETF energy density functional as a phenomenological approximation for the ground-state energy density functional. This Skyrme-ETF functional is known ${ }^{6}$ ) to be an excellent realization of the density functional for the average ground state energy - without 
shell effects, see ref. ${ }^{17}$ ) - at least for medium and heavy nuclei. It might be seen whether modifications are necessary if it is to be used also for small nuclei.

For the sake of transparency and simplicity we restrict our calculations to spherical $N=Z$ nuclei disregarding also the Coulomb effects. The ETF4 energy functional including gradients of the density up to the fourth order then simplifies appreciably ${ }^{18}$ ). A variational ansatz for the density $\rho$ of a spherical nucleus of the following form is used,

$$
\rho(r)=\rho_{0}\left\{\frac{1+\cosh \left(R_{0} / \alpha\right)}{\cosh \left(R_{0} / \alpha\right)+\cosh (r / \alpha)}\right\}^{\gamma} .
$$

This so-called symmetrized Woods-Saxon density ${ }^{19}$ ) (for the case $\gamma=1$ ) is obtained by multiplying a standard Woods-Saxon density with the form obtained by its reflection at the origin $r=0$. A Woods-Saxon density would not be a sufficiently general variational ansatz for the calculation of desaturation in small nuclei since the ansatz must a priori guarantee a vanishing first derivative $\mathrm{d} \rho / \mathrm{d} r$ of $\rho$ at the origin. It might be important for the description of desaturation to start from a density with a realistic functional form just at the origin since desaturation effects are intimately connected with the degree of freedom of the bulk density ${ }^{5}$ ). The parameters $\alpha$ and $\gamma \neq 1$ describe the surface thickness and the skewness of the surface profile, respectively. The parameters $\rho_{0}, R_{0}, \alpha$ and $\gamma$ are all $A$-dependent and interrelated by the fact that the density $(2.1)$ has to be normalized to a given particle number $A$. Thus, there are three independent variational parameters in the ansatz (2.1).

For medium heavy and heavy nuclei, the difference between densities of the form (2.1) and usual Woods-Saxon (or Fermi) densitics taken to the power $\gamma$, such as used in ref. ${ }^{6}$ ), is totally negligible. Thus, all the results and conclusions of the variational Skyrme-ETF4 calculations of ref. ${ }^{6}$ ) - to which we refer for the technical details - can be immediately taken over also when using the density profiles (2.1). Significant differences in the results arise only in very light systems $(A \leqslant 10)$ and will be mentioned in sect. 3 when discussing the results. The skewness $y \neq 1$ of the density profiles (symmetrized or not) plays, however, a quantitative role also for medium and heavy systems [see ref. ${ }^{6}$ ) and figs. 1,2 below].

The results of the Skyrme-ETF4 calculations are then compared with the results obtained from the slab model prescription of ref. ${ }^{13}$ ). This model allows to separate desaturation effects from others. It is described in detail in ref. ${ }^{13}$ ), and we here only describe its basic input. Desaturation in the model of a nuclear slab can be studied by comparing density profile and specific energy of a slab with those of the semi-infinite system. From these desaturation effects in plane nuclear slabs with limiting surfaces in one direction, one comes to desaturation in spherical nuclei with limitations in all three dimensions by multiplying the desaturation effects in slabs by a factor of three. In such a way one obtains from the density profiles of slabs of various thicknesses the profiles $\rho=\rho(r)$ of spherical nuclei where the particle 
number $A$ is obtained by integrating the profile $\rho(r)$ and where the corresponding energy densities are obtained by inserting this function $\rho(r)$ into the Skyrme-ETF functional. The quantities obtained in this way for a spherical nucleus are compared with those obtained by the variational approach; the validity of the slab model can thus be checked explicitly. Another check of the slab model prescription for spherical nuclei consists in the comparison of the resulting energies with those obtained from a mass formula whose leading coefficients, including at least a curvature term, are found from semi-infinite nuclear matter calculations. Hereby only curvature (i.e. sphericity) effects in the coefficient $a_{\mathrm{c}}$ of the $A^{1 / 3}$ contribution and in the coefficient $a_{0}$ of the $A^{0}$ contribution are considered. Compression effects cannot be included in the slab model approach since one starts from a plane system. However, all terms in $A^{1 / 3}, A^{0}, A^{-1 / 3}$ etc. which follow explicitly from the sphericity of the nucleus are included. The advantage of the slab model calculation now is that it allows to separate the pure desaturation effects from the sphericity effects which hide the desaturation terms in the variational calculation with the density ansatz (2.1).

For the slab model calculation we used the Skyrme-ETF2 energy functional renormalized in such a way that the neglect of fourth-order gradient terms is compensated in an optimal way by readjusting two of the ETF2 coefficients. Within the ETF2 approach one obtains an Euler-Lagrange equation for the slab density which can be easily integrated. The ETF2 energy functional has the following functional structure

$$
\mathscr{E}[\rho]=h(\rho)+A \frac{\rho^{\prime 2}}{\rho}+B \rho^{\prime 2}+C \rho^{\prime 2} \rho .
$$

Here, the function $h(\rho)$ is given by

$$
h(\rho)=\frac{3}{5}\left(\frac{3 \pi^{2}}{2}\right)^{2 / 3} \frac{\hbar^{2}}{2 m} \frac{m}{m^{*}}(\rho) \rho^{5 / 3}+\frac{3}{8} t_{0} \rho^{2}+\frac{1}{16} t_{3} \rho^{2+1 / 6},
$$

with

$$
\frac{m}{m^{*}}=1+\beta \rho
$$

All parameters $t_{0}, t_{3}, A, B, C, \beta$ can be found e.g. in ref. ${ }^{6}$ ) for the SkM and SkM* interactions. B and C depend on the density due to the $\rho$-dependence of the effective mass $m^{*}$. The renormalization of the ETF2 parameters to reproduce fourth-order effects means multiplication of the Weizsäcker term $A$ by a factor of 1.4 [see ref. ${ }^{20}$ )] and a reduction of the spin-orbit strength appearing in the parameter $C$ by a factor of $\frac{2}{3}$, since the fourth-order spin-orbit terms are known to cancel about $50 \%$ of the second-order spin-orbit terms ${ }^{6,21}$ ). For comparison we also used the parameterization of Treiner and Krivine ${ }^{7}$ ). The results did not change appreciably. 
The Euler-Lagrange equation following from the condition of the specific slab energy to be stationary reads as follows [see ref. ${ }^{13}$ ) for derivation],

$$
\rho^{\prime 2}=\frac{h(\rho) / \rho-\varepsilon_{\mathrm{F}}}{A+B \rho+C \rho^{2}} \rho^{2} .
$$

The Lagrange multiplier $\varepsilon_{\mathrm{F}}$ controls the particle number per unit area. The dependence of the effective mass $m^{*}$ on the density $\rho$ does not change the simple structure of eq. (2.2), and the parameters $A, B$ and $C$ may be functions of the density $\rho$ without changing the result (2.4).

\section{Numerical results}

\subsection{GENERALIZED LIQUID DROP MODEL EXPANSION OF THE ENERGY}

The parameters $\rho_{0}, \alpha$ and $\gamma$ in the variational ansatz (2.1) for the density which minimize the total energy of a spherical $N=Z$ nucleus for the SkM*-ETF4 functional (neglecting the Coulomb terms) are displayed as functions of the mass number $A$ in fig. 1. Appreciable desaturation effects in the nuclear density are apparent below $A \sim 30$ where the central density $\rho_{0}$ decreases remarkably from its saturated value

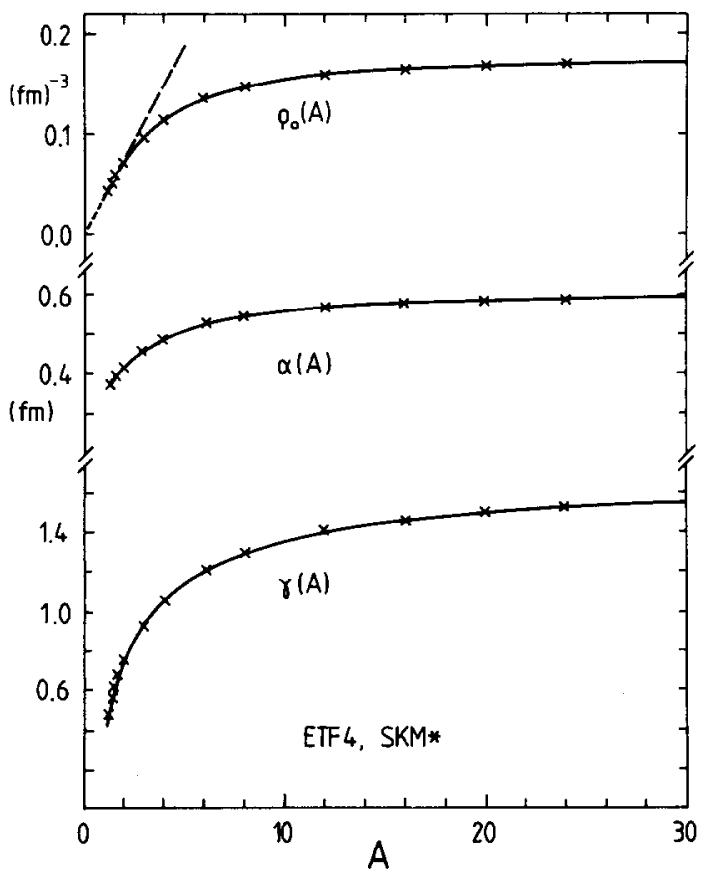

Fig. 1. The parameters $\rho_{0}, \alpha$ and $\gamma$ of the symmetrized Woods-Saxon density (2.1) obtained for SkM* in the variational calculation. 
$\left(\approx \rho_{\infty}\right)$ in such a way that small nuclei are "blown up." Also the diffuseness parameter $\alpha$ and the skewness parameter $\gamma$ of the nuclear density differ appreciably from their asymptotic values if $A \leqslant 30$. The surface becomes steeper and more symmetric (for $A>4)$ as $A$ decreases.

The characteristic quantity

$$
\frac{\delta \rho}{\rho_{\infty}}=\frac{\rho_{0}-\rho_{\infty}}{\rho_{\infty}},
$$

which measures the difference between the central density $\rho_{0}=\rho_{0}(A)$ of a nucleus and the saturated nuclear matter value $\rho_{\infty}$, is plotted in fig. 2 versus $A^{-1 / 3}$ by the solid line. The deviation of this curve from the dashed-dotted straight line, given by $\left(\delta \rho / \rho_{\infty}\right)_{\mathrm{DM}}=\left(6 a_{\mathrm{s}} / K_{\infty}\right) A^{-1 / 3}$ in the droplet model $\left.{ }^{1}\right)$, characterizes the desaturation effect on the central density. $\left(A^{-2 / 3}\right.$ corrections to $\left(\delta \rho / \rho_{\infty}\right)_{\mathrm{DM}}$ are small $\left.{ }^{6,7}\right)$, see eq. (3.5) below.)

The curve $\delta \rho / \rho_{\infty}\left(A^{-1 / 3}\right)$ is rather sensitive to the skewness degree of freedom $y$ of the density profile $\rho(r)$ eq. (2.1). In fact, a similar curve is shown in ref. ${ }^{6}$ ) (see fig. 11 there), which was obtained with simple Fermi-function profiles [with $\gamma=1$,

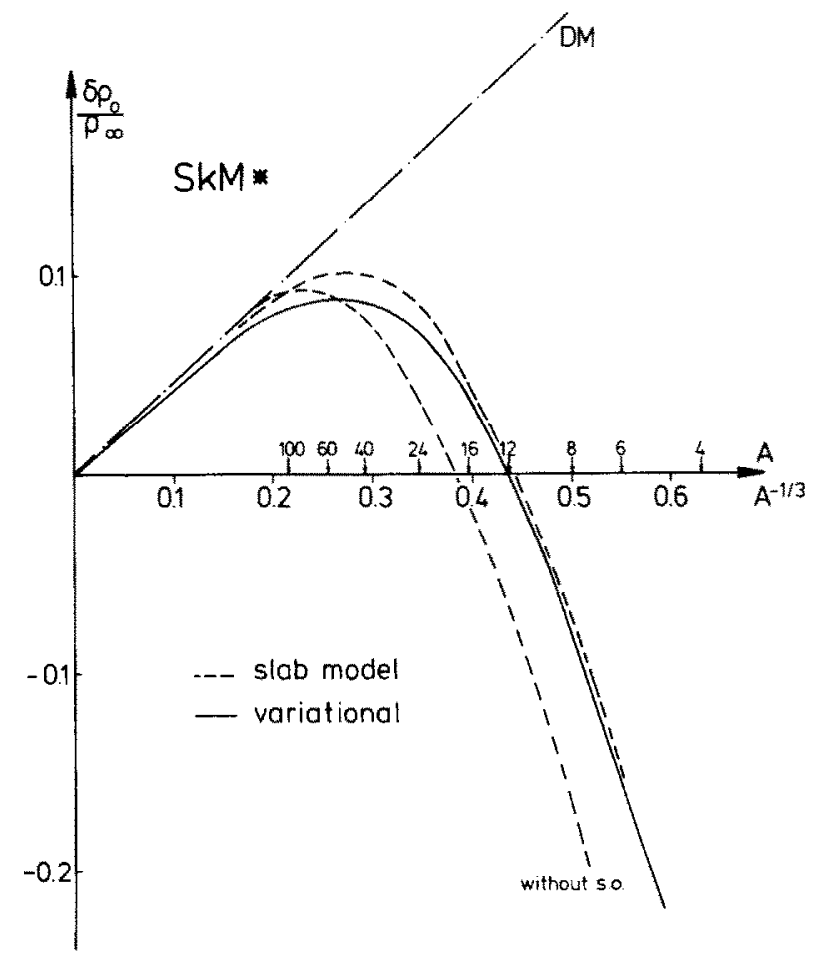

Fig. 2. The relative density change $\delta \rho / \rho_{\infty}(3.1)$ as a function of $A$ and $A^{-1 / 3}$, respectively. The full curve represents the result of the variational calculation. The broken curves follow for ETF2 from the slab model approach with and without the inclusion of the spin-orbit interaction part. 
which was not explicitly stated in ref. $\left.\left.^{6}\right)\right]$. The maximum there occurred at $A \cong 125$ corresponding to $\delta \rho / \rho_{\infty} \cong 0.04$, thus leading to a much smaller compression effect. The variation of $\gamma$ thus allows for a stronger squeezing of the central density due to the surface tension. The effect on the energy of this degree of freedom is much smaller and amounts to a lowering by only $\sim 2-5 \mathrm{MeV}$ for $A \leqslant 240$. The symmetrization of the profile $\rho(r)$ eq. (2.1) (guaranteeing that $\rho^{\prime}(0)=0$ ), although theoretically important, has practically little influence on the quantitative results. (In fact, with Fermi functions to a variable power $\gamma$ we found values of $\rho(0), \alpha$ and $\gamma$ which agree with the SWS ones shown in fig. 1 to within less than $2 \%$ for all $A \geqslant 10$. The curve $\delta \rho / \rho_{\infty}\left(A^{-1 / 3}\right)$ also agrees with that on fig. 2 to within a few percent in the region of its maximum $(A \geqslant 30)$. The variational energies obtained with the two types of density profiles even agree within less than $0.03 \mathrm{MeV}$ for all $A \geqslant 2$.) Note that the curve $\delta \rho / \rho_{\infty}\left(A^{-1 / 3}\right)$ is very similar to that found in ref. $\left.{ }^{5}\right)$ with a slightly different interaction (SkM) and a phenomenologically readjusted second-order ETF energy density.

In fig. 3 the $\delta \rho / \rho_{\infty}$ curve is displayed over a larger range of $A$-values such that the hypothetical limit $A \rightarrow 0$ can be studied. From its very definition (3.1) and the extrapolation $\rho_{0} \rightarrow 0, \delta \rho / \rho_{\infty}$ must be -1 in this limit. Now, the variational calculation can only be carried down to $A \approx 1.4$ since no energy minimum could be found for smaller values of $A$. (I.e. systems with $A \leqslant 1.4$ are unbound.) However, from a linear extrapolation of the values $\rho_{0}(A)$ given in fig. 1 down to zero for $A=0$, the dotted line of fig. 3 follows as an extrapolation of the calculated $\delta \rho / \rho_{\infty}$ curve. This supplement fits smoothly to the curve obtained for $A>1.4$ by the variational calculation. It should be noted that the $A=2$ value for $\delta \rho / \rho_{\infty}$ obtained in the

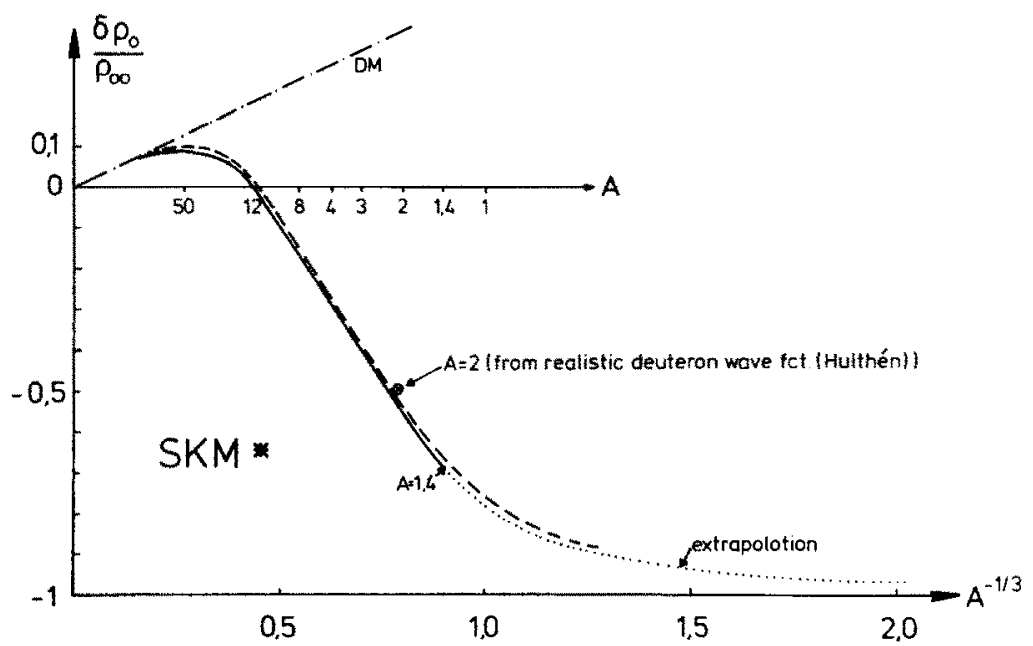

Fig. 3. $\delta p / \rho_{\infty}$ from the variational calculation (full curve) and from the slab model approach (broken curve). 
variational method agrees well with the semi-empirical value obtained by fitting a Hulthén deuteron wave function as well as possible to empirical data ${ }^{22}$ ). Even the variational ETF energy obtained for $A=2(E=-2.55 \mathrm{MeV})$ is not unreasonable. That is a hint that the SkM ${ }^{*}$-ETF energy density functional is a reasonable realization of the exact energy density functional even for small values of $A$.

We tried to reproduce the numerical energies $E=E(A)$, obtained by the variational method, by a generalized LDM expansion in terms of $A^{-1 / 3}$,

$E_{\mathrm{LDM}}=a_{\mathrm{v}} A+a_{\mathrm{s}} A^{2 / 3}+a_{\mathrm{c}}^{*} A^{1 / 3}+a_{0}^{*} A^{0}+a_{-1} A^{-1 / 3}+a_{-2} A^{-2 / 3}+a_{-3} A^{-1}$.

From infinite and semi-infinite nuclear matter calculations the values of $a_{\mathrm{v}}$ and $a_{\mathrm{s}}$ are known to be ${ }^{6}$ ) (for the $\mathrm{SkM}^{*}$ force) $a_{\mathrm{y}}=-15.77624 \mathrm{MeV}$ and $a_{\mathrm{s}}=17.219 \mathrm{MeV}$; $a_{\mathrm{c}}^{*}$ consists of the pure curvature part $a_{\mathrm{c}}$ and the compressibility part $-2 a_{\mathrm{s}}^{2} / K_{\infty}$, adding up to ${ }^{6}$ ) $a_{\mathrm{c}}^{*}=10.235 \mathrm{MeV}$. [Note that in the semi-infinite limit, the SWS profiles (2.1) become identical to the Fermi functions to the power $\gamma$ used in ref. ${ }^{6}$ ) to obtain these LDM parameters.]

With these three leading LDM parameters $a_{\mathrm{v}}, a_{\mathrm{s}}$ and $a_{\mathrm{c}}^{*}$ given $a$ priori, we now attempt to fit the generalized LDM expansion eq. (3.2) into our numerical results for $E(A)$. This is most easily done by plotting the quantity $\Delta E$ defined by

$$
\Delta E=E(A)-a_{\mathrm{v}} A-a_{\mathrm{s}} A^{2 / 3}-a_{\mathrm{c}}^{*} A^{1 / 3}
$$

versus $A^{-1 / 3}$. The corresponding values of $\Delta E$ are shown in fig. 4 by the crosses. We see that $-11 \mathrm{MeV} \leqslant \Delta E \leqslant-8 \mathrm{MeV}$ for all $A \geqslant 4$. Thus, already adding a constant

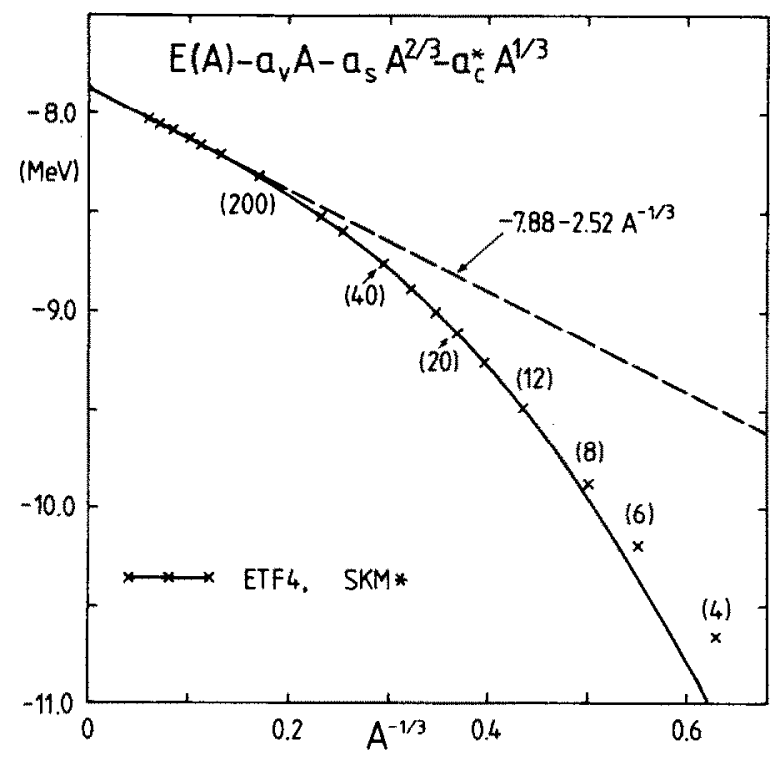

Fig. 4. The difference $\Delta E$ eq. (3.3) as a function of $A^{-1 / 3}$ for the variational calculation using $\mathrm{SkM}^{*}$ in the ETF4 approximation. The continuous curve is obtained from eq. (3.2) with the parameters given in the text. 
term $\tilde{a}_{0} \cong-8.8 \mathrm{MeV}$ to the LDM mass formula would allow to reproduce the variational ETF4 energies to within less than $1 \mathrm{MeV}$ for all $A \geqslant 8$. The correct asymptotic value of $a_{0}$, however, is found from the linear fit to $\Delta E\left(A^{-1 / 3}\right)$ shown by the dashed straight line in fig. 4 , yielding $a_{0}^{*}=-7.88 \mathrm{MeV}$ and $a_{-1}=-2.52 \mathrm{MeV}$. With these two extra terms, the validity of the LDM formula with a $1 \mathrm{MeV}$ accuracy is extended down to $A=4$. The procedure can now be continued by making a linear fit (in $A^{-1 / 3}$ ) to $A^{2 / 3}$ times the difference between the numerical values of $E$ and the dashed line in fig. 4. This yields the values $a_{-2}=0.99 \mathrm{MeV}$ and $a_{-3}=-8.55 \mathrm{MeV}$. Feeding these parameters into the extended LDM formula eq. (3.2) leads to the solid curve in fig. 4 which reproduces the exact variational energies to within less than $0.2 \mathrm{MeV}$ for $A \geqslant 6$.

It would be meaningless to extend this procedure further, adding higher order terms in $A^{-1 / 3}$ to eq. (3.2). Already at this stage, the parameters start to diverge: $\left|a_{-3}\right|>\left|a_{-2}\right|$. This would remain so even if higher terms were included. Thus, the necessity to include contributions to $E$ different from the LDM type shows up for $A \leqslant 6$. This will be attempted in the remainder of this section. It is also obvious that eq. (3.2) cannot be expected to work for very small $A$, since the energy $E_{\mathrm{LDM}}$ (3.2) is singular for $A=0$. We consider it as quite remarkable that the mass formula (3.2) does perform so well down to values of $A \cong 10$ where desaturation of the densities clearly is present. This is not in contradiction with the fact that the desaturation effects on the density $\rho_{0}$ already become important for $A \leqslant 40$ (see figs. 2 and 3 ). [We recall the general theorem proved in ref. ${ }^{5}$ ) that the down turn of $\delta \rho / \rho_{\infty}\left(A^{-1 / 3}\right)$ cannot be explained from an energy expansion of the form (3.2) ${ }^{\star}$.] Since the energy is a stationary quantity, it is affected only to second order by the desaturation effects in $\rho_{0}$, and thus "feels" them only for much smaller systems.

\subsection{SEPARATION OF DESATURATION EFFECTS}

We now want to compare these numerical results with the slab model calculation described in sect. 2 which, on its turn, is able to separate the pure desaturation effects from the LDM terms. The slab model method cannot take into account specific compression effects in the density, e.g. one cannot describe the squeezing of the bulk density by the surface tension and the effect of curvature and surface compressibility on the central density. Thus, we approximate - starting from the slab model value of $\left(\delta \rho / \rho_{\infty}\right)_{\text {des }}$ which is a pure desaturation effect - the value of $\delta \rho / \rho_{\infty}$ for spherical nuclei by the sum of $\left(\delta \rho / \rho_{\infty}\right)_{\text {des }}$ and the droplet model $\left.{ }^{1}\right)$ value $\left(\delta \rho / \rho_{\infty}\right)_{\mathrm{DM}}$

$$
\frac{\delta \rho}{\rho_{\omega}}=\left(\frac{\delta \rho}{\rho_{\omega}}\right)_{\mathrm{DM}}+\left(\frac{\delta \rho}{\rho_{\omega}}\right)_{\mathrm{des}} .
$$

* It can, however, be reproduced if the Taylor expansion of $\delta \rho / \rho_{\infty}\left(A^{-1 / 3}\right)$ is resummed in a particular Padé-like form, see ref. $^{6}$ ). 
In an extended LDM picture the droplet model value can be improved by including a curvature correction term proportional to $A^{-2 / 3}\left[\right.$ refs. $\left.^{6,7}\right)$ ],

$$
\left(\frac{\delta \rho}{\rho_{\infty}}\right)_{\mathrm{DM}}=\frac{6 a_{\mathrm{s}}}{K_{\infty}} A^{-1 / 3}+\eta A^{-2 / 3} \text {. }
$$

It can be shown using the minimum principle for the energy that the expressions given for $\eta$ in refs. ${ }^{6,7}$ ) are identical. As outlined in ref. ${ }^{13}$ ) the ansatz (3.4) cannot work for very small values of $A$ since in principle coupling terms would have to be included, otherwise there is no guarantee that the asymptotic limit $\delta \rho / \rho_{\infty}=-1$ for $A=0$ is obtained. In fig. 2 we have inserted dashed curves which display the $\delta \rho / \rho_{\infty}$ values obtained from the ansatz (3.4) for the SkM*-ETF2 functional with and without the inclusion of spin-orbit parts. The slab model curve agrees reasonably well with that of the variational approach. It can be seen that the spin-orbit interaction diminishes the desaturation effect; analogously, it reduces the surface thickness ${ }^{21}$ ). (Note that the LDM limit for $A \rightarrow \infty$ is different for the two broken curves in fig. 2 since the LDM coefficients $a_{\mathrm{s}}$ are different.) The slab model prediction for $\delta \rho / \rho_{\infty}$ is also compared with the variational values in fig. 3 over a larger range of $A$-values than in fig. 2 . There is a good overall agreement down to very small values of $A$ of both methods. From fig. 3 the desaturation contribution $\left(\delta \rho / \rho_{\infty}\right)_{\text {des }}$ can be seen to be of the exponential form $\exp \left(-\mu A^{1 / 3}\right)$ only in the region of intermediate values of $A$ down to around 10. As shown in table 1 the effect of desaturation on the central density of a nucleus becomes larger than three percent below $A \approx 38$ for the $\mathrm{SkM}^{*}$ interaction. Neglecting spin-orbit effects would increase this critical value of $A$ to $A \approx 60$.

We now analyze the energies $E(A)$ obtained in the slab model approach for spherical nuclei in order to extract exponential terms. The slab model prescription for the calculation of nuclear energies can be checked by comparing these energies with those obtained from a mass formula of the type (3.2) where the coefficients are calculated independently from the semi-infinite nuclear matter limit. We find

TABLE 1

The DM parameters $a_{s}, a_{\mathrm{c}}$ and $a_{0}$ as calculated in ETF2 approximations from semi-infinite nuclear matter properties. $\bar{a}_{0}$ is the slab model limiting value for $a_{0}$ (see text). In the last two columns the critical $A$-values are given below which there is a desaturation effect of more than $3 \%$.

\begin{tabular}{|c|c|c|c|c|c|c|c|}
\hline & & \multirow[b]{2}{*}{$a_{\mathrm{s}}[\mathrm{MeV}]$} & \multirow[b]{2}{*}{$a_{\mathrm{c}}[\mathrm{MeV}]$} & \multirow[b]{2}{*}{$a_{0}[\mathrm{MeV}]$} & \multirow[b]{2}{*}{$\bar{a}_{0}[\mathrm{MeV}]$} & \multicolumn{2}{|c|}{$\begin{array}{l}3 \% \text { desaturation } \\
\text { effect on }\end{array}$} \\
\hline & & & & & & $\begin{array}{l}\text { central } \\
\text { density }\end{array}$ & $\begin{array}{l}\text { total } \\
\text { energy }\end{array}$ \\
\hline SkM & incl. s.o. & 16.82 & 11.61 & -16.72 & -15.9 & $A=32$ & 3 \\
\hline SkM & without s.o. & 18.05 & 13.08 & -23.78 & -22.17 & 53 & 5 \\
\hline SkM* & incl. s.o. & 17.44 & 12.40 & -19.13 & -18.20 & 38 & 3.5 \\
\hline SkM* & without s.o. & 16.62 & 13.85 & -26.44 & -24.56 & 60 & 5.5 \\
\hline
\end{tabular}


for the SkM*-ETF2 energy functional $a_{\mathrm{v}}=-15.77 \mathrm{MeV}, a_{\mathrm{s}}=17.44 \mathrm{MeV}$. For the pure curvature coefficient $a_{\mathrm{c}}$ we obtain $12.4 \mathrm{MeV}$ and for the $a_{0}$ coefficient we use $-18.2 \mathrm{MeV}$. The coefficients of the higher order terms $a_{-1} A^{-1 / 3}$ etc. in the mass formula cannot be given by closed expressions in terms of semi-infinite nuclear matter quantities. This truncated mass formula reproduces the energies of the slab model prescription excellently for $A>40$. Below $A=30$ the difference $\Delta E$ between the energies $E_{\mathrm{sl}}$ of the slab model approach and $E_{\mathrm{LDM}}$ from the truncated mass formula is positive and increasing from around $0.3 \mathrm{MeV}$ to $2.3 \mathrm{MeV}$ for $A=4$. This discrepancy, however, cannot be taken as a hint of (small) desaturation effects which taken alone would be negative. The difference $\Delta E$ first of all shows the higher order terms $a_{-1} A^{-1 / 3}$ etc.

Another check for the validity of the slab model prescription is given - as described in ref. ${ }^{13}$ ) - by the comparison of $a_{0}$ as calculated using eq. (3) of ref. ${ }^{13}$ ) analytically from semi-infinite nuclear matter quantities with the slab model value $\bar{a}_{0}$, defined by

$$
\bar{a}_{0}=E_{\mathrm{sl}}(A)-\left(a_{\mathrm{v}} A+a_{\mathrm{s}} A^{2 / 3}+a_{\mathrm{c}} A^{1 / 3}\right),
$$

for large values of $A(A \geqslant 100)$. As table 1 shows, the agreement between $a_{0}$ and $\bar{a}_{0}$ is satisfactory.

After these checks of the reliability of the slab model to treat sphericity as well as desaturation correctly, we now use it to extract the pure desaturation energy in nuclei. The procedure is carried out in the following way. For the determination of the density of a hypothetical "saturated" nucleus we cut out density profiles from the semi-infinite saturated nuclear matter system and insert these profiles $\rho(r)$ into the energy density functional in order to obtain the energies of spherical nuclei whose mass numbers follow by integrating $\rho(r)$. Then we compare these values $E_{\text {sat }}(A)$ with the values $E(A)$ obtained from the slab model for minimum energy nuclei. Fig. 5 shows that both curves coincide practically for values of $A \geqslant 6$ which means that no desaturation effect in the energy can be seen above $A=6$. More quantitatively we obtained (see table 1) a three-percent desaturation effect on the energy below $\boldsymbol{A}=3$ for the SkM*-ETF2 functional with spin-orbit parts included. Without spin-orbit effects desaturation is more important as can be seen from fig. 5 and table 1. Below $A=6$ the curve for the energies $E$ and $E_{\text {sat }}$ splits up into two branches in such a way that the energies of minimum energy nuclei are smaller than those of hypothetical saturated nuclei. It is obvious that the pure desaturation energy must come out to be negative. This follows also from the general argument that any degree of freedom for changing the density is used to lower the energy.

In fig. 6 we have plotted the desaturation energy per particle as a function of the mass number $A$ for the SkM* interaction. We tried to fit this curve by an exponential ansatz of the form $a_{1} \exp \left(-\gamma_{1} A^{1 / 3}\right)$ and also by an ansatz $a_{2} A^{-1 / 3} \exp \left(-\gamma_{2} A^{1 / 3}\right)$ in such a way that the points 1 and 2 of the curve are placed on the exponential curves also. In both cases the approximations were bad fits (the dashed line is the first exponential fit). For a more limited region of $A$-values, e.g. $4 \leqslant A \leqslant 6$, an 


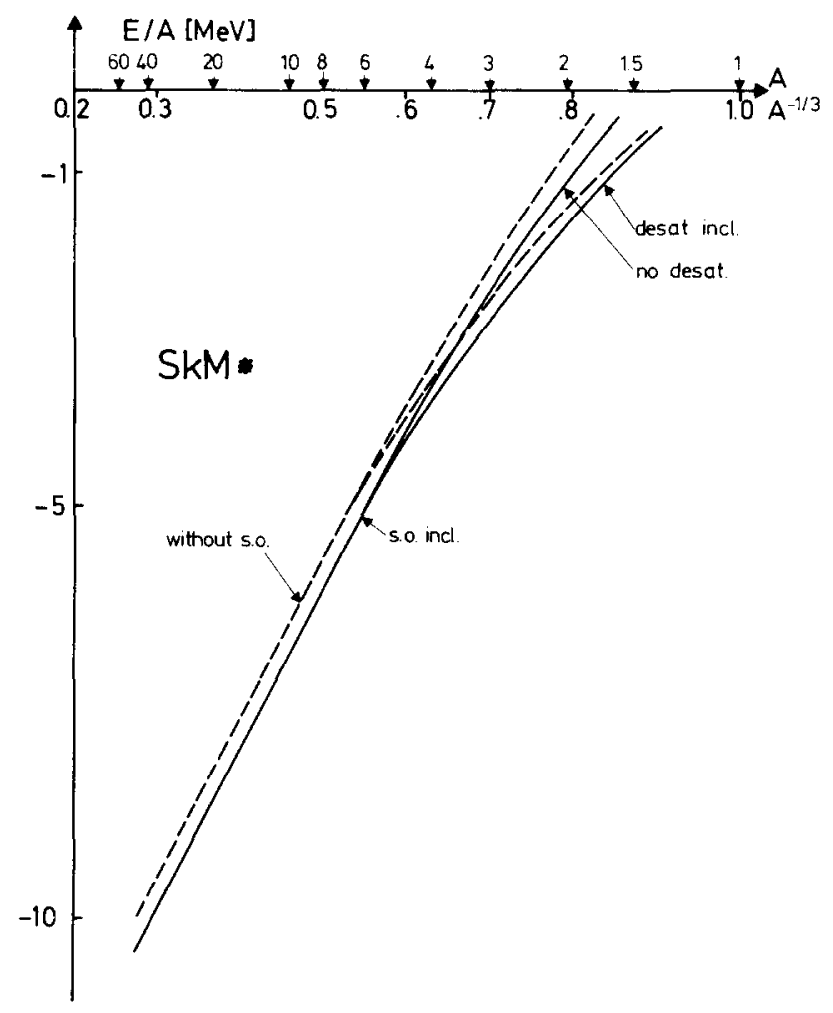

Fig. 5. The slab model prediction for the energy per particle as a function of $A$ and $A^{-1 / 3}$, respectively, for the SkM* interaction in the ETF2 approximation if desaturation effects are included and excluded. The dashed curves are calculated without the spin-orbit term.

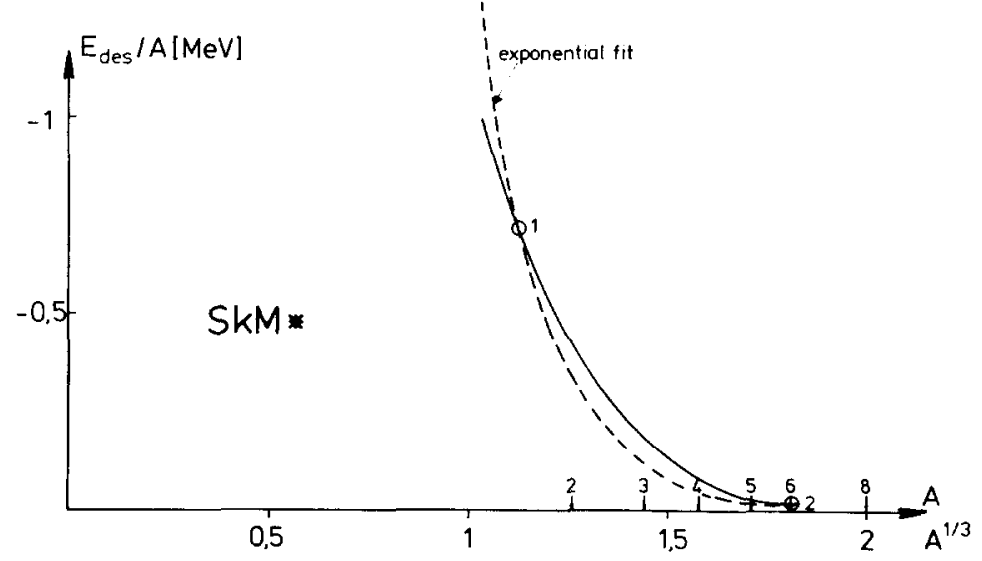

Fig. 6. The desaturation energy per particle following from the slab model approach using the SkM* interaction. 
exponential approximation would fit the curve more reasonably. For a larger region $1.5 \leqslant A \leqslant 6$, a good fit which practically coincides with the continuous curve for $E_{\text {des }} / A$ of fig. 6 can be obtained from a sum of two exponentials given by

$$
E_{\text {des }} / A \approx\left(16-34 A^{-1 / 3}\right) \exp \left(-2.6 A^{1 / 3}\right) .
$$

This reflects the result obtained in the appendix showing the importance of terms coupling LDM and desaturation effects, e.g. volume-exponential and surfaceexponential terms.

The curve for the difference $\Delta E=E_{\mathrm{sl}}-E_{\mathrm{LDM}}$, as a function of $A^{-1 / 3}$, has a parabolic shape above $A \approx 8$. Below $A \approx 8$ it exhibits a bending which is removed if the desaturation effects (see fig. 6) are subtracted from $E_{\mathrm{sl}}$. We conclude therefore that the pure LDM part of the mass formula is improved if terms $a_{-1} A^{-1 / 3}$ and $a_{-2} A^{-2 / 3}\left(a_{-1} \approx-2.8 \mathrm{MeV}, a_{-2} \approx 11.7 \mathrm{MeV}\right)$ are added to the truncated mass formula. The exponential desaturation part then shows up below $A \approx 8$ as a bending.

As outlined above, the slab model prescription cannot account for the compression effect in spherical nuclei coming from the pressure on the bulk from the surface tension. Similarly, this approach does not include a term coupling compression and desaturation. Such a term must therefore be analyzed using the energies $E(A)$ following from the variational calculation. In lowest order this term must have the form $A^{1 / 3} \exp (\cdots)$. (Note that also the pure curvature-exponential term, which is included in the slab model approach, is of this form. This contribution was shown above to be small.) Such a term coupling compression and desaturation should show up if one compares the slab model values and the energies of the variational calculation for spherical nuclei. However, first one has to add to the slab model encrgies the corrections taking care of the DM compression effects. One knows ${ }^{1,6}$ ) how $a_{\mathrm{c}}$ and $a_{0}$ have to be modified to take into account these compression contributions; we called the modified coefficients $a_{\mathrm{c}}^{*}$ and $a_{0}^{*}$, respectively. Adding these compression corrections to the slab model energies the corrected energies coincide with those of the variational calculation within less than $1 \mathrm{MeV}$ for $A<80$ down to $A \approx 4$. This shows that the compression-exponential encrgy, which must be identical with the remaining discrepancy between the two energy values, is small. In consistency with this conclusion we recall also the fact, shown in fig. 4 and discussed above, that a mass formula of liquid drop model type is able to reproduce all energies down to $A \approx 4$ without the inclusion of exponential terms, if the expansion is extended to include terms down to $a_{-3} A^{-1}$. Exponential contributions only show up below $A \approx 4$ if at all.

As outlined in the appendix there is a volume-exponential term which has the opposite sign of the pure DM volume term; also the surface exponential term in (A.15) is opposite in sign compared to the pure DM surface term. The case of a slab is obtained from (A.15) by setting the DM compression term in ( $\Lambda .1)$ equal to zero, i.e. $\alpha=0$. Therefore the slab model cannot take into account the compressionexponential coupling term of eq. (A.15), and we had to get information about this 
term by a very careful analysis using the energies of spherical nuclei following from the variational calculation of sect. 3 . We saw no indication that this term is as large as exponential terms found in ref. ${ }^{11}$ ). It should be noted that a term coupling pure curvature and exponential effects is included in the slab model calculation and found to be negligible as well as the other exponential terms, coupling volume, surface and exponential effects.

Introducing new degrees of freedom, first the compression degree of freedom of the DM picture with a surface squeezing the bulk, and secondly the other degree of freedom of rearranging the density due to desaturation, always leads to a decrease in magnitude of the corresponding pure liquid drop terms. Compression reduces the $A^{1 / 3}$ term and also the $A^{0}$ term. Desaturation reduces in magnitude the $A^{1}$ volume and the $A^{2 / 3}$ surface term. Thus, we suppose that the general structure of the mass formula could be reproduced by the ansatz

$$
E=a_{\mathrm{v}}(A) A+a_{\mathrm{s}}(A) A^{2 / 3}+a_{\mathrm{c}}^{*}(A) A^{1 / 3}+a_{0}^{*}(A) A^{0}+\cdots,
$$

with all $a$-coefficients reaching their constant DM values for $A>A_{\text {crit }}$ and with the limit $a(A=0)=0$.

\section{Summary and conclusions}

We have presented variational calculations for energies and densities of hypothetical nuclei with $N=Z$ and without Coulomb interaction. We used the extended Thomas-Fermi (ETF) density functionals in connection with realistic effective nucleon-nucleon interactions of the Skyrme type. This model does not reproduce shell effects and is therefore an ideal tool for studying the average properties of nuclei. For medium and heavy nuclei, these have been shown ${ }^{6}$ ) to reproduce rather accurately the average parts of microscopical Hartree-Fock results. On the other hand, the density variational method can also be extended to very light systems and yields reasonable densities and energies even for such light systems as $\boldsymbol{A}=2$. This model thus also allows to study the desaturation effects which are the main object of this paper.

We have seen that an appreciable desaturation of the density (i.e. decreasing of the central density $\rho_{0}$ ) sets in for $A \leqslant 40$ (for the $\mathrm{SkM}^{*}$ force including spin-orbit interaction; without the latter for $A \leqslant 60$ ). In ref. ${ }^{5}$ ) a general theorem was proven, saying that the downturn of $\rho_{0}\left(A^{-1 / 3}\right)$ cannot be obtained - within the density variational formalism - from a "leptodermous" or liquid drop model (LDM) type expansion of the energy in terms of $A^{-1 / 3}$. The desaturation of the densities, seen here for $A \leqslant 40$, must therefore be linked to "non-leptodermous" terms, and the question arises to what extent these contribute to the energies.

We have therefore studied the generalization of the LDM expansion of the energy and found that a mass formula including terms down to $A^{-1}$ - see eq. (3.2) reproduces the variational energies - which do contain the non-leptodermous 
contributions - with an accuracy of $0.05 \mathrm{MeV}$ for all $A \geqslant 10$ and, with an error of $\leqslant 1 \mathrm{MeV}$, even down to $A=4$. It must therefore be concluded that the non-leptodermous terms, although showing up in the densities for $A \leqslant 40$, contribute negligibly to the energies for $A \geqslant 10$. The reason for this is simply that the energy is a variational quantity and therefore is affected only to second order in small density changes.

Our conclusion is that there is no reason for $A \geqslant 10$ to include non-leptodermous terms in the mass formula, such as e.g. the exponential terms considered in refs. ${ }^{11,12}$ ). Even for very light systems with $4 \leqslant A \leqslant 10$, their contributions to the energy are so small that they cannot possibly be disentangled from the shell effects, whose amplitudes are of the order of several $\mathrm{MeV}$, in the real nuclear binding energies.

We have substantiated these conclusions by a careful analysis of the variational ETF results in terms of the slab model ${ }^{13}$ ) which allows explicitly to separate the pure desaturation terms from the LDM terms.

Parts of this paper were worked out when one of the authors (W.S.) was visiting the Los Alamos National Laboratories. He is grateful to Dr. J.R. Nix for his hospitality and useful discussions and hints. The authors also thank Dr. M. Farine for his interest in this work.

\section{Appendix}

\section{EXPONENTIAL TERMS IN THE ENERGY EXPRESSION}

We start from the ansatz

$$
\frac{\delta \rho}{\rho_{\infty}}=\alpha A^{-1 / 3}-\beta \exp \left(-\gamma A^{1 / 3}\right)
$$

for the relative change $\delta \rho / \rho_{\infty}$ of the central density $\rho_{0}^{A}$ of a nucleus with mass number $A$ with respect to the infinite nuclear matter value $\rho_{\infty}$. The functional form of the ansatz (A.1) can be motivated by the analytical slab calculation of ref. ${ }^{13}$ ) performed with a schematic energy density, if an expansion of the expression $\delta \rho / \rho_{\infty}$ of this ref. is made around the critical value $\boldsymbol{A}_{\text {crit }}$ where the desaturation effect is still small. Also the numerical curves for $\delta \rho / \rho_{\infty}$ are very well fitted in an intermediate range of $A$-values by such a functional form and suitable positive parameters $\alpha$ and $\beta$. The ansatz (A.1), however, cannot be valid down to very small $A$-values. Here, terms coupling DM and exponential effects appear in order to guarantee the limit $\delta \rho / \rho_{\infty}$ to be -1 for $A \rightarrow 0$.

The aim of the following consideration now is to extract terms in the expression for the energy $E_{A}$ of a finite nucleus resulting from the exponential correction in eq. (A.1). One has to insert (A.1) into the energy expression and make use of as many criteria as possible which guarantee the energy to be stationary around the exact density $\rho_{A}(r)$. These criteria will take care for the correction terms in the 
energy to be of second order due to the stationarity of the energy with respect to density changes. Thus, one expects the exponential terms in the energy to be of the form $\exp \left(-2 \gamma A^{1 / 3}\right)$.

We denote the energy density functional by

$$
\mathscr{E}=\mathscr{E}\left[\rho_{A}(r)\right]
$$

Then the specific energy $E / A$ in the bulk as a function of the bulk density $\rho_{0}^{A}$ is approximated by

$$
\varepsilon_{\mathrm{F}}^{A}=\frac{E}{A}\left(\rho=\rho_{0}^{A}\right)=a_{\mathrm{v}}^{\infty}+\frac{K_{\infty}}{18}\left(\frac{\delta \rho}{\rho_{\infty}}\right)^{2}+\cdots
$$

with $a_{\mathrm{v}}^{\infty}$ being the energy per particle in saturated nuclear matter and $K_{\infty}$ the incompressibility. The energy $E_{A}$ is split up into a volume part and a surface part,

$$
E_{A}=\varepsilon_{\mathrm{F}}^{A} \int \rho_{A}(r) \mathrm{d}^{3} r+\int\left\{\mathscr{E}\left[\rho_{A}\right]-\varepsilon_{\mathrm{F}}^{A} \rho_{A}(r)\right\} \mathrm{d}^{3} r
$$

Using (A.1) and (A.3) the volume part is rewritten as

$$
E_{A}^{\mathrm{vol}}=a_{\mathrm{v}}^{\infty} A+\frac{K_{\infty}}{18}\left\{\alpha^{2} A^{-2 / 3}-2 \alpha \beta A^{-1 / 3} \exp \left(-\gamma A^{1 / 3}\right)+\beta \exp \left(-2 \gamma A^{1 / 3}\right)\right\} A
$$

For the evaluation of the surface part of eq. (A.4) we introduce the equivalent sharp radius $R_{\text {sh }}^{A}$,

$$
R_{\mathrm{sh}}^{A}=r_{0}^{A} A^{1 / 3}, \quad r_{0}^{A}=\left(\frac{3}{4 \pi \rho_{0}^{A}}\right)^{1 / 3}
$$

Then, we substitute

$$
r=R_{\mathrm{sh}}^{\mathrm{A}}+u
$$

rewriting the integral in eq. (A.4),

$$
E_{A}^{\mathrm{surf}}=4 \pi\left(R_{\mathrm{sh}}^{A}\right)^{2} \int_{-R_{\mathrm{sh}}^{A}}^{\infty}\left\{\mathscr{E}\left[\rho_{A}\right]-\varepsilon_{\mathrm{F}}^{A} \rho_{A}\right\} \mathrm{d} u+\cdots
$$

The neglected part contains the pure curvature energy, higher order terms and, especially, a possible term of the form $A^{1 / 3} \exp (\cdots)$, coupling pure curvature and exponential effects, which can be included in the slab model approach. Introducing now the abbreviation $\sigma_{A}\left(\rho_{0}^{A}\right)$ for the integral in (A.8), we have

$$
E_{A}^{\text {surf }}=4 \pi\left(\frac{3}{4 \pi}\right)^{2 / 3} A^{2 / 3} \frac{\sigma_{A}\left(\rho_{0}^{A}\right)}{\left(\rho_{0}^{A}\right)^{2 / 3}} .
$$


The next step is intended to extract those terms in (A.9), which are of first order in $\delta \rho / \rho_{\infty}$,

$$
E_{A}^{\text {surf }}=\left.4 \pi\left(\frac{3}{4 \pi}\right)^{2 / 3} A^{2 / 3} \frac{\mathrm{d}}{\mathrm{d} \rho_{0}^{A}}\left(\frac{\sigma_{A}\left(\rho_{0}^{A}\right)}{\left(\rho_{0}^{A}\right)^{2 / 3}}\right)\right|_{\rho_{0}^{A}=\rho_{\infty}} \delta \rho+\cdots
$$

One obtains

$$
E_{A}^{\text {surf }}=4 \pi\left(\frac{3}{4 \pi}\right)^{2 / 3} A^{2 / 3}\left\{-\frac{2}{3} \frac{\sigma_{\infty}}{\rho_{\infty}^{5 / 3}}\right\} \delta \rho,
$$

if the $\dot{\sigma}=0$ theorem is used following from saturation of nuclear matter at $\rho_{\infty}$ and the stationarity of the surface tension $\sigma_{\infty}$ of a plane nuclear matter surface with fixed bulk density $\rho_{\infty}\left[\right.$ ref. $\left.\left.^{1}\right)\right]$. The neglection of the correction terms to $\mathrm{d} \sigma_{A} / \mathrm{d} \rho_{0}$ means neglection of terms of the order $A^{-1 / 3}$ and exponential terms. Finally, we insert (A.1) into (A.11),

$$
E_{A}^{\text {surf }}=-\frac{1}{9} \alpha^{2} K_{\infty} A^{1 / 3}+\frac{1}{9} \alpha \beta K_{\infty} \exp \left(-\gamma A^{1 / 3}\right) A^{2 / 3},
$$

where we used that $\alpha=6 a_{\mathrm{s}} / K_{\infty}$ in first order DM. One sees by adding the volume part $E_{A}^{\mathrm{vol}}$ to (A.12) that the minimum principles for the energy, used up to here, first let emerge the standard compression energy and in addition guarantee the linear exponential terms to vanish.

The second-order contribution to $E_{A}^{\text {surf }}$ can be evaluated only approximately, since we can take into account the minimum principle for the energy only schematically. The second-order contribution to $\sigma\left(\rho_{0}^{A}\right) /\left(\rho_{0}^{A}\right)^{2 / 3}$ is written as

$$
\left.\frac{\sigma\left(\rho_{0}^{A}\right)}{\left(\rho_{0}^{A}\right)^{2 / 3}}\right|_{\text {sec. ord. }}=\left.\frac{1}{2}(\delta \rho)^{2}\left\{\frac{\ddot{\sigma}_{A}}{\left(\rho_{0}^{A}\right)^{2 / 3}}-\frac{4}{3} \frac{\dot{\sigma}_{A}}{\left(\rho_{0}^{A}\right)^{5 / 3}}+\frac{10}{9} \frac{\sigma_{A}}{\left(\rho_{0}^{A}\right)^{8 / 3}}\right\}\right|_{\rho_{\infty}} .
$$

Again making use of the $\dot{\sigma}=0$ theorem and using the schematic model of ref. ${ }^{23}$ ) for $\ddot{\sigma}_{A}$, we end up with the following expression for the second-order term in the surface part $E_{A}^{\text {surf }}$ of the energy,

$$
\left(E_{A}^{\text {surf }}\right)_{\text {sec.ord. }}=-\frac{22}{9} 4 \pi\left(\frac{3}{4 \pi \rho_{\infty}}\right)^{2 / 3} \sigma_{\infty}\left(\frac{\delta \rho}{\rho_{\infty}}\right)^{2} A^{2 / 3} .
$$

Collecting now all terms, the final expression for $E_{A}$ is found to be of the form,

$$
\begin{aligned}
E_{A}= & \mathrm{DM} \text { part }+{ }_{18}^{1} K_{\infty} \beta^{2} \exp \left(-2 \gamma A^{1 / 3}\right) A \\
& -44\left\{\alpha^{2}-2 \alpha \beta \exp \left(-\gamma A^{1 / 3}\right) A^{1 / 3}+\beta^{2} \exp \left(-2 \gamma A^{1 / 3}\right) A^{2 / 3}\right\} .
\end{aligned}
$$

The validity of this expression is limited to the region of $A$ where the ansatz (A.1) for $\delta \rho / \rho_{\infty}$ with fixed parameters $\alpha, \beta$ and $\gamma$ is working, i.e. only above $A \approx 20$, where desaturation in the encrgy is still negligible. Thus, it is not allowed to extrapolate (A.15) to small values of $A$. The minimum principle for the energy could not be taken fully into account in all steps leading to (A.15), which makes the term, 
coupling curvature-compression and exponential terms, doubtful. Including an exponential term of this structure in a finite range droplet mass formula fit to empirical masses, Moeller and $\mathrm{Nix}^{24}$ ) found a negligible decrease of the root-mean square deviation. The coefficients found in such a fit for this term also show that it is probably of second-order as all the other exponential terms. For values of $A \approx 6$, where desaturation becomes appreciable, eq. (A.15) can only be retained if all coefficients are renormalized (compare the end of sect. 3.2). Comparing eq. (3.7) with (A.15) reduction factors of about 15 are seen for volume-exponential and surface-exponential terms. The signs of the corresponding terms as well as (approximately) their ratio, however, are the same.

\section{References}

1) W.D. Myers and W.J. Swiatecki, Ann. of Phys. 55 (1969) 395

2) J.M. Pearson, Phys. Lett. 91B (1980) 325

3) F. Tondeur, J.M. Pearson and M. Farine, Nucl. Phys. A394 (1983) 462

4) F. Tondeur and D. Berdichevsky, J. of Phys. G12 (1986) 33

5) J. Treiner, W.D. Myers, W.J. Swiatecki and M.S. Weiss, Nucl. Phys. A452 (1986) 93

6) M. Brack, C. Guet and H.-B. Håkansson, Phys. Reports 123 (1985) 275

7) J. Treiner and H. Krivine, Ann. of Phys. 170 (1986) 406

8) H.J. Krappe and J.R. Nix, Proc. 3rd Int. Conf. on physics and chemistry of fission, Rochester 1973, vol. I (IAEA, Vienna, 1974) p. 159

9) W. Stocker, Nucl. Phys. A324 (1979) 21

10) B. Grammaticos, Ann. of Phys. 126 (1980) 450; 139 (1982) 1

11) P. Möller, W.D. Myers, W.J. Swiatecki and J. Treiner, Proc. 7th Conf. on atomic masses and fundamental constants, AMCO-7, ed. O. Klepper (THD, Darmstadt, 1985) p. 457

12) A.K. Dutta, J.-P. Arcoragi, J.M. Pearson, R.H. Behrmann and M. Farine, Nucl. Phys. A454 (1986) 374

13) M. Farine and W. Stocker, Nucl. Phys. A459 (1986) 117

14) T.H.R. Skyrme, Phil. Mag. 1 (1956) 1043

D. Vautherin and D.M. Brink, Phys. Rev. C5 (1972) 626

15) D. Gogny, Nucl. Phys. A237 (1975) 399

16) J.P. Hohenberg and W. Kohn, Phys. Rev. 136 (1964) B864; M. Levy and J.P. Perdew, Density functional methods in physics, ed. R.M. Dreizler and J. da Providencia, NATO ASI Series, vol. 123 (Plenum, New York, 1985) p. 11

17) M. Brack and P. Quentin, Nucl. Phys. A361 (1981) 35

18) Y.H. Chu, B.K. Jennings and M. Brack, Phys. Lett. 68B (1977) 40;

C. Guet and M. Brack, Z. Phys. A297 (1980) 247

19) B. Grammaticos, Z. Phys. A305 (1982) 257

20) M. Brack,Density functional methods in physics, ed. R.M. Dreizler and J. da Providencia, NATO ASI Series, vol. 123 (Plenum, New York, 1985) p. 331

21) B. Grammaticos and A. Voros, Ann. of Phys. 129 (1980) 153

22) G.E. Brown and A.D. Jackson, The nucleon-nucleon interaction (North-Holland, Amsterdam, 1976)

23) M. Brack and W. Stocker, Nucl. Phys. A388 (1982) 230

24) J.R. Nix and P. Möller, private communication (1986) 